\title{
LES LIVRES DE SYDRAC L'ÉVOLUTION D'UN DIALOGUE ENCYCLOPÉDIQUE
}

\author{
Savoir, c'est questionner, répondit Reb Mendel. \\ Que tirerons-nous de ces questions [...] qui nous entraîneront \\ à poser d'autres questions [...] ? dit le second disciple. \\ La promesse d'une nouvelle question, répondit Reb Mendel. \\ Edmond Jabès, Le livre des questions ${ }^{1}$.
}

Des centaines de fois, le roi Boctus pose questions sur questions au philosophe et « astronomien » Sydrac, qui ne se lasse pas de répondre. La soif de vouloir savoir est inextinguible. Le livre volumineux dans lequel Boctus fait transcrire le dialogue en est le reflet fidèle. Il nous est parvenu en deux versions, une version courte comportant 613 questions et réponses, et une version longue qui en présente le double (1227 questions et réponses). Elles ont eu toutes deux beaucoup de succès, comme en témoignent les nombreux manuscrits (plus de 70) et les imprimés (10), qui, de 1486 à 1537, ne laissent survivre que le texte de la version longue. Elle n'arrête pas de provoquer d'autres questions et questionnements chez les lecteurs modernes, comme le montre le conte Les oreilles du comte de Chesterfield et le Chapelain Goudman de Voltaire, qui reprend le dialogue de Sydrac à sa façon ${ }^{2}$, ou encore un lecteur au siècle suivant, qui en 1836 comble la lacune de son exemplaire — un imprimé de 1516 auquel manquent "quelques feuillets ${ }^{3}$ - avec de nouvelles «demandes ou

1. E. Jabès, Le livre des questions, Paris, 1963, p. 125.

2. Éd. A. Beuchot, CEuvres de Voltaire, avec préfaces, avertissements, notes, etc., Paris, 1829, t. 34, p. 423-451. C'est à Cosmin Popa (Genève) que nous devons l'identification de ce document de réception, cf. la publication qu'il va faire paraître à ce sujet sous le titre "Sydrac et Sidrac : du dialogue encyclopédique à la conversation philosophique » et dans laquelle il analyse en détail « l'irrespectueuse intertextualité » (p. 4) que pratique Voltaire avec sa source, Le Livre de Sydrac.

3. Voir le texte complet de la notice inscrit au début de l'imprimé (Paris, BNF, Rés. M. $\left.Y^{2} 122\right)$ : « Avis au lecteur. Comme tu le remarqueras en ce volume quelques 
interrogations »; s'il n'y ajoute pas toujours de réponse, c'est qu'il aimerait « laisser carrière plus vaste à ton imagination, lecteur, mon ami » ${ }^{4}$. Comme en passant, cette petite note définit ce qui distingue radicalement le dialogue entre Boctus et Sydrac de questionnements modernes pour lesquels «questionner c'est construire un chemin " ${ }^{5}$. À l'ouverture, au plaisir de pouvoir chercher soi-même la réponse, qui ne sera que « la promesse d'une nouvelle question », le Livre opposait la fermeture, par la fermeté des réponses dont aucune ne sera jamais mise en question par Boctus. Le roi, heureux de trouver en Sydrac celui qui pouvait lui expliquer enfin ce que personne jusque-là jamais ne « dire li seust » (Prol. 1, §4), est satisfait des réponses et a hâte de poser une nouvelle question qui oriente souvent le dialogue vers un nouveau thème.

Autant le dialogue médiéval entre le roi et le philosophe présente un monde clair et transparent, où même la Trinité se laisse entrevoir par lécanomantie pour convertir le souverain mécréant, autant son auteur s'entoure de mystères. Il se cache derrière la figure fictive de Sydrac, construite sur le modèle de Daniel et prophète comme lui, médiateur d'un savoir dont il a hérité avec le livre que Dieu fit dicter à Noé, et cet effacement au profit de son invention est si total que nous ignorons tout de lui. Les essais pour combler cette lacune, allant d'abord d'un fantaisiste "médecin arabe de Cordoue converti au christianisme " ${ }^{6}$ à

feuillets se trouvent perdus par l'injure du temps. On pouvait y suppléer à l'aide d'un autre exemplaire complet, mais il s'en rencontre si difficilement (la majeure partie de l'édition ayant péri dans l'espace de plus de trois siécles) il m'a semblé que je pouvais joindre aux questions et réponses du bon-homme Sydrach quelques demandes ou interrogations aux quelles il est vrai je ne prendrai pas toujours la peine de répondre pour plusieurs raisons et surtout pour laisser carrière plus vaste à ton imagination, lecteur mon ami. J'ai ajouté à la fin du volume la vie de Madame Saincte Marguerite morceau ancien et peu commun et quelques autres bonnes choses qui font que ce volume a un commencement et une fin $»$.

4. Si un autre témoignage de 1866 parle de «L'œuvre récréatif du vieux sage Sidrac ", ce qualificatif ne semble pas résulter d'une connaissance intime du texte, mais s'explique plutôt par une allusion au titre du volume que l'auteur anonyme de la « Fantaisie bibliographique » a dû avoir dans sa bibliothèque (cf., par ex., le titre de l'imprimé de Galliot du Pré, Paris, 1531 : « Mil IIII vingtz et quatre demandes, avec les solutions et responses a touz propos, œuvre curieux et moult récréatif selon le sage Sidrach ») : J. S., Fantaisie bibliographique, Paris, 1866 ; texte accessible en ligne http://www. textesrares. com/ fantai.htm.

5. Cf. M. Heidegger, « Die Frage nach der Technik », dans Id., Vorträge und Aufsätze, Pfullingen, 1954, p. 13-44, ici p. 13 : «Das Fragen baut an einem Weg ».

6. Auguste François Louis Scipion de Grimoard-Beauvoir du Roure de Beaumont-Brison, Analectabiblion, ou Extraits critiques de divers livres rares, oubliés ou peu connus, tirés du cabinet du marquis D. $\mathrm{R}^{* * *}$, Paris, 1836, t. 1, p. 231-234 («Sydrach le grant philosophe, Fontaine de toutes sciences», article dédié à un 
«Sydrac, philosophe hébreu » ${ }^{7}$, que Leclerc croyait pouvoir identifier comme juif du «midi de la France ${ }^{8}$, n'ont plus avancé depuis les propositions d'Ernest Renan et de Gaston Paris - l'auteur pourrait être un Jehan Pierre de Lyon, nommé dans le premier prologue - et depuis celle de Langlois, suivant laquelle l'auteur aurait vécu en Terre Sainte et aurait composé la Fontaine de toutes sciences vers la fin du XIII ${ }^{\mathrm{e}}$ siècle.

À constater combien la recherche fait voyager le mystérieux inconnu entre les cultures qui se croisent autour de la Méditerranée, on comprend la force d'attraction du premier prologue, qui retrace le voyage du texte entre les rives de ce bassin et à travers les siècles jusqu'en 1243, où il aurait été apporté d'Antioche à Tolède. Cette fiction est authentifiée par la citation de noms de personnages historiques, introduits - pour parler avec T. L. Burton - « merely as a name-dropping exercise, to lend an air of authenticity to a spurious history ${ }^{9}$. D'autres textes de l'époque procèdent de la même façon et citent aussi dans leur prologue Frédéric II ou son philosophe et astrologue Theodore ${ }^{10}$.

$\mathrm{Au}$ lieu de vouloir percer le mystère savamment construit par un inconnu à l'imagination particulièrement fertile et d'essayer d'identifier quelqu'un qui a tout fait pour ne pas l'être, il nous semble plus prometteur d'avancer dans l'analyse de ce que nous avons - l'œuvre dans ses deux versions - , pour comprendre ce vaste texte dans son ensemble, et de le comprendre comme tel.

Cela nous semble d'autant plus nécessaire que l'œuvre a provoqué depuis les débuts des recherches modernes de vives réactions négatives, qui n'ont guère profité au désir de s'en approcher de façon approfondie. La consternation face aux défauts du Livre, à son "grossier charlatanisme » et à son « manque absolu de talent et de goût » ${ }^{11}$, à sa puérilité (" perhaps

imprimé du $\mathrm{XvI}^{\mathrm{e}} \mathrm{s}$.), ici p. 231. Sous le même titre, le marquis bibliophile a fait paraître en 1837 un deuxième tome non moins riche de cette œuvre intéressante.

7. Le Roux de Lincy, « Mélanges bibliographiques », dans Bulletin du bibliophile, t. 7 (1836), p. 239-40, ici p. 239. Voir aussi P. Paris qui parle du « juif Sidrac» dans sa description du Livre d'après le manuscrit Paris, BNF, fr. 762, Les manuscrits de la Bibliothèque du Roi, t. 6, Paris, 1845, p. 24-31, ici p. 24.

8. V. Leclerc, "L'Image du monde et autres enseignements », dans Histoire littéraire de la France, t. 23, Paris, 1856, p. 294.

9. T. L. Burton, Sidrak and Bokkus. A parallel-text edition from Bodleian Library, MS Laud. Misc. 559 and British Library, MS Lansdowne 793, Oxford, t. 1, 1998 [EETS, 311], p. xxiv.

10. Voir Ch.-V. Langlois, La vie en France au moyen âge, t. 3, La connaissance de la nature et du monde, Paris, 1926, p. 198-275, ici p. 206-208.

11. E. Renan-G. Paris, " La Fontaine de toutes sciences du philosophe Sidrach », dans Histoire littéraire de la France, t. 31, Paris, 1893, p. 285-318, ici p. 317. 
the most puerile encyclopaedia of the Middle Ages ») ${ }^{12}$ et à la « détestable logorrhée d'homme sans culture littéraire ni autre » qu'il présenterait ${ }^{13}$, cette consternation ne s'est pas atténuée avec le temps. Segre encore, face à la «mediocrità dell'opera » et aux répliques « tanto futili», aurait aimé pouvoir au moins «cogliere nell'autore il sorriso di chi risponde con affettata solennità ai quesiti più idioti », mais n'en trouve pas ${ }^{14}$.

En ce qui concerne l'autre grand défaut du texte que serait son « désordre ", Ernest Renan et Gaston Paris avaient déjà terminé leur article en appelant de leurs vœux, pour le « livre, un des plus mal composés d'une époque où l'art de bien faire un livre était assez peu connu [...] » ${ }^{15}$, « [u]ne édition critique, dans laquelle on essayerait de le rapprocher autant que possible de la forme où l'auteur l'écrivit » ${ }^{16}$. Et Cesare Segre espère de même qu'une édition critique pourrait « attenuare » le « disordine con cui si susseguono le domande » dans ce mélange confus (« farragine ») que représenterait La fontaine de toutes sciences ${ }^{17}$.

Espoir qui s'est avéré vain : l'analyse de la riche tradition manuscrite et des imprimés, entreprise dans la préparation de notre édition, ne laisse pas

12. G. Sarton, Introduction to the History of Science, Washington, 1927-1931, t. 2, p. 589-591, ici p. 590. Voir aussi Ch.-V. Langlois, op. cit., p. 214, pour lequel le Livre de Sydrac dépasse "en puérilité » toutes les encyclopédies de l'époque. S. Treanor, dans sa thèse de 1939, arrive à la conclusion suivante : "For any one interested today in the illiterate thought of the Middle Ages, - its superstitions, naïveté, utter fatuousness, puerility and stupidity, the work is a happy hunting ground » (Le Roman de Sydrac, Fontaine de toutes sciences, Chapel Hill, p. xxiii). Voir aussi Y. Lefèvre, qui parle de fréquentes « considérations saugrenues et puériles ", dans L'Elucidarium et les Lucidaires. Contribution, par l'histoire d'un texte, à l'histoire des croyances religeuses en France au moyen age, Paris, 1954, p. 324.

13. Ch.-V. Langlois, op. cit., p. 214.

14. «Accoppiamenti (forse) giudiziosi », dans C. Segre éd., Linguistica e filologia. Omaggio a Benvenuto Terracini, Milano, 1968, p. 255-278, ici p. 258. Ce qu'il trouve au contraire, « è piuttosto un certo ben calcolato interesse ai fatti sessuali ; ingrediente indispensabile per qualunque bestseller antico o moderno » (p. 258) Ce rapprochement élargit outre mesure un sujet marginal qui est noyé dans l'immensité du dialogue. Voir aussi, chez V. Minervini, Il 'libro di Sidrac', versione catalana, Bari, 1982, p. ix, la même insistance sur les « argomenti prediletti » du Livre de Sydrac: « i problemi della generazione [...] o l'ossessivo interesse per il peccato di lussuria », et de nouveau récemment encore chez F. Duval: Lectures françaises de la fin du moyen âge. Petite anthologie commentée de succès littéraires, Genève, 2007, p. 220 : « Du point de vue thématique, Sidrac témoigne d'un intérêt particulier pour la sexualité et la reproduction, ainsi que pour l'astrologie ».

15. Voir, de même, dans la transcription de la première moitié du Livre d'après le manuscrit BNF, fr. 1160, le constat catégorique de S. Treanor, op. cit., p. xxii : « No similar work of the Middle Ages surpasses the Sidrac in lack of plan ».

16. E. Renan-P. Paris, op. cit., p. 317-318.

17. C. Segre, op. cit., p. 258 et 266. 
de doutes : le prétendu « désordre » est l'ordre du Livre de Sydrac. Constat qui peut paraître paradoxal, mais qui contient la clé du problème, comme le révèle une approche détaillée de l'œuvre. Grâce à elle, nous aimerions faire voir dans ce qui suit que, si l'ordre $e t$ le désordre déterminent ce long texte, c'est qu'ils se fondent sur une conception qui les organise et dans laquelle ils trouvent leur équilibre spécifique ${ }^{18}$.

\section{Cadrages}

Au départ, tout est ordre dans La Fontaine de toutes sciences. Le savoir qui y est exposé est doublement encadré par des récits. Un prologue qui retrace l'histoire du texte, de sa composition dans les temps reculés de Sydrac jusqu'au milieu du XIII siècle, est suivi d'une longue narration qui raconte quelles circonstances auraient provoqué jadis le long dialogue du philosophe avec le roi de Boctorie ; au bout de centaines de questions et de réponses, elle se termine par la conversion définitive de Boctus et la solution du problème qui l'avait empêché jusque-là de venir à bout de son voisin Garab, roi des Indes.

Cette fiction qui entend procurer à l'œuvre un rang tout à fait exceptionnel (le savoir transmis reposerait sur le livre, dicté par un ange, que Sydrac aurait hérité de son ancêtre Noé) nécessite des efforts accrus d'authentification, pour lesquels l'auteur inconnu a recours à différents systèmes de légitimation qui se renforcent mutuellement. Les deux récits reconstituent, d'un bout à l'autre de la chaîne événementielle, une continuité des plus rassurantes. La répartition sur deux récits s'explique par les formes du savoir sur lesquelles chacun des deux est basé, le premier prologue sur celle du savoir scientifique, le second sur celle du savoir narratif. Le premier vise la structure de l'histoire fictive, le second son fond, avec la genèse du conflit entre le roi mécréant et le philosophe " astronomien».

Le premier prologue se présente comme le résultat d'un examen auquel un groupe d'experts (« plusors maistres et clers ») à Tolède aurait soumis le Livre de Sydrac en $1243^{19}$. C'est une entrée en matière astucieusement

18. Voir à ce sujet aussi notre article, "Wissensvermittlung in Frage und Antwort. Der enzyklopädische Lehrdialog Le Livre de Sidrac », dans H. BrunnerN. R. Wolf éd., Wissensliteratur im Mittelalter und in der frühen Neuzeit. Bedingungen, Typen, Publikum, Sprache, Wiesbaden, 1993 [Wissensliteratur im Mittelalter. Schriften des SFB 226 Würzburg/Eich-stätt, 13], p. 26-35, en part. p. 29-30.

19. La version longue du Livre sera citée dans ce qui suit d'après notre édition, Sydrac le philosophe, Le livre de la fontaine de toutes sciences. Edition des enzyklopädischen Lehrdialogs aus dem XIII. Jahrhundert, Wiesbaden, 2000 [Wissensliteratur im Mittelalter. Schriften des SFB 226 Würzburg/Eichstätt, 34 ; Ruhe, ed. cit.], ici p. $3, \S 12$. Nous citons la version courte d'après la transcription du manuscrit 
montée, peu conforme à ce qu'on attendrait d'un «sot» pour lequel Langlois prenait l'auteur ${ }^{20}$. Le comité de Tolède a formulé son constat suivant les règles propres à l'accessus ad auctores, c'est-à-dire que les experts donnent, dans l'ordre traditionnel, les réponses aux questions qu'il fallait poser dans l'introduction d'un texte prévu pour la lectio, allant du quis (vita auctoris) et du cur (causa scribendi) au quid sit titulus operis ${ }^{21}$. Dans le procès-verbal qui clôt leur prologue et dans lequel ils révèlent leur identité et indiquent la date de leur examen, ils expliquent où ils sont intervenus dans le Livre - ils ont ajouté quelques gloses 22 - et surtout où ils ne l'ont pas fait :

il ne porent acorder de metre en cest livre les chapitres qui touchent a une raison les uns aprés les autres, mais il acorderent de laisser les chapitles l'un aprés l'autre ensi comme il sont escrit en cest livre tels comme li roi Boctus les requist au sage philosophe Sydrac. ${ }^{23}$

$\overline{\text { Paris, BNF, fr. 1160, faite par Sapelo Treanor, op. cit., pour la première moitié du }}$ manuscrit (Treanor, ed. cit.) et par William McFall Holler pour la seconde (Le livre de Sydrac, Fontaine de toutes sciences, folios 57-112, Chapel Hill, 1972 ; Holler, ed. cit.). Le texte présenté dans les thèses américaines n'est pas toujours correct et se révèle à maints endroits difficile à comprendre. De plus, le manuscrit BNF, fr. 1160, utilisé de préférence dans la recherche depuis Langlois, n'est pas sans poser problème : souvent le scribe a sauté par mégarde des parties du texte; les questions 97-103 manquent, ainsi qu'un folio entre f. 32 et 33 ; de plus, les questions posées par Boctus ont été laissées en blanc pour les questions 149-177 (p. 145-152 de l'éd. Treanor).

20. Ch.-V. Langlois, op. cit., p. 214.

21. Voir, pour plus de détails, notre analyse, «Die Legitimierung des Wissens. Der enzyklopädische Lehrdialog Le Livre de Sydrac", dans A. Zucker éd., L'encyclopédisme antique et médiéval : innovation et pédagogie. Colloque de Nice, novembre 2010 [sous presse].

22. «Et si ont aucunes choses glosees de cest livre par la conoissance des choses qui furent avant de nos et par l'art de phylosophie » (éd. Ruhe, p. $4, \S 12$; cf. pour la version courte éd. Treanor, p. 8). Dans le texte du Livre, ces éventuelles gloses ne sont pas identifiées comme telles. Mais il y a trois questions qui sont introduites par une rubrique dans la tradition d'un nota bene marginal, ce qui suggère au lecteur une telle intervention : question 32 « Cest chapitres est encontre les Greus » (cette question manque dans la version courte) ; 435 (version courte 297, éd. Holler, p. 33-34) "Cest chapitre est encontre toutes les nations qui dient que non est espurgeeur »; 648 (version courte 491, éd. Holler, p. 140-142) « Cest chapitre si est contre toutes les nascions qui dient pour quoi les Frans ne donent au pueple du cors que li prestres reçoit et du sanc que il reçoit avuecques le cors ». Pour ces questions et leur contexte du Contra errores Graecorum de Thomas d'Aquin et du concile de Lyon de 1274, voir notre article de 1993, op. cit., p. 31.

23. Éd. Ruhe, p. 3-4, § 12 (cf. pour la version courte éd. Treanor, p. 8). 
C'est l'ordre du texte qui posait problème, car il était en contradiction évidente avec l'attente professionnelle. À la fin de la discussion, le groupe d'experts a décidé de ne pas intervenir, en réorganisant le texte selon la structure systématique conforme aux règles, et de tout laisser tel quel. La raison en est le respect pour un genre spécifique, le dialogue, et pour un document venant d'une autre époque. Puisque Boctus aurait fait transcrire ses questions et les réponses de Sydrac dans l'ordre de leur conversation, les « maistres et clers » de Tolède préfèrent garder intacte cette forme qui reflèterait fidèlement le vécu (« l'un aprés l'autre [...], tels comme li roi Boctus les requist »). La construction fictive du premier prologue sert ainsi de caution à la structure de l'œuvre, dont l'ouverture et la mobilité seraient les traces de l'oralité.

Le second prologue sert de caution à une structure qui se place à l'opposé. Il tire du savoir narratif sa légitimation, mise en scène dans la «forme par excellence de ce savoir» consensuel qu'est le récit ${ }^{24}$. L'histoire de la rencontre entre Sydrac et Boctus s'appuie sur des modèles intertextuels importants pour l'époque, comme le livre de Daniel, le roman de Merlin et le recueil d'apologues Kalila et Dimna ; ce qui les relie est ce qui est aussi au centre du conflit entre le roi et le philosophe : ce sont des histoires de conversion.

La religion comme «métarécit» de l'époque constitue la "perspective d'ensemble » du Livre de Sydrac ${ }^{25}$. Cette fonction se réfléchit de façon particulièrement marquante dans certaines parties de l'œuvre. La discussion sur la religion de Sydrac, à laquelle celui-ci veut gagner le roi idolâtre, encadre le long dialogue et introduit l'ordre systématique propre à la source qui y est mise à contribution, c'est-à-dire la traduction française la plus répandue de l'Elucidarium d'Honorius Augustodunensis. De larges extraits de ce manuel, choisis le plus souvent dans l'ordre de la source, constituent le point de départ (« Qui est ton dieu ? ») ${ }^{26}$ et le point d'arrivée du dialogue (la venue de l'Antéchrist, le Jugement Dernier), avec deux points d'appuis importants entre les deux bornes ${ }^{27}$.

24. Jean-François Lyotard, La condition postmoderne. Rapport sur le savoir, Paris, 1979, p. 38.

25. J.-F. Lyotard, op. cit., p. 7 et 39.

26. Éd. Ruhe, p. $42, \S 17$ (version courte : éd. Treanor, p. 57). Pour la source, voir Lucidaire I 2, éd. M. Türk, 'Lucidaire de grant sapientie'. Untersuchung und Edition der altfranzösischen Übersetzung 1 des 'Elucidarium' von Honorius Augustodunensis, Tübingen, 2000 [Beihefte zur $\mathrm{ZrPh}$, 307], p. 212.

27. Les citations commencent déjà au milieu du second prologue (Lucid. I 2 et I 3 , éd. Ruhe, p. $42, \S 17$ et p. $45, \S 26$; cf. pour la version courte éd. Treanor, p. 57 et 62 ) et continuent dans les questions 1 à 48 (version courte 1-36 : voir Lucid. I 10 à II 33), de même qu'elles dominent également la fin du Livre, de la question 1153 (version courte 591) jusqu'à la question 1187 (version longue 613), qui reprennent la fin du 
La partie qui précède le point d'arrivée est la plus systématique de l'œuvre. Elle est précédée d'une série de questions sur les bêtes (436-450 ; version longue 586-601) et d'une collection de recettes de médecine (451478 ; version longue 602-631), qui sont comme une annonce de ce qui suit. Une rubrique, unique en son genre dans l'œuvre, marque la césure :

Nos avons demandé et entendu de l'esperituel et dou corporel et si volons savoir des amis de Dieu qui furent d'Adam jusques a Noé ${ }^{28}$.

La partie finale du Livre qui est annoncée ainsi commence par une suite de questions extraites du Lucidaire (481-506 ; version longue 637-666). Au centre se trouve un vaste traité astrologique, qui est suivi d'un lapidaire (509-533 ; version longue 1065-1091) et d'une autre collection de recettes de médecine, concentrées sur l'emploi des « herbes précieuses » (534-583; version longue 1092-1142), avant que ne se termine le Livre avec la série d'extraits du Lucidaire, déjà cités, sur la fin du monde.

Parmi les traités, celui sur l'astrologie a une place à part non seulement par son étendue, mais surtout aussi à cause d'une introduction étoffée qui en souligne l'importance. Elle se présente sous la forme d'un récit, qui rappelle par maints détails les récits contenus dans les deux prologues. Après Adam, auquel Dieu permit de connaître cet art, Japhet, fils de Noé, l'aurait appris d'un ange. Il aurait couché par écrit ce savoir pour le transmettre à la postérité, et ce livre "de l'un a l'autre vint en nostre poöir » ${ }^{29}$, formule qui résume en quelques mots ce que le premier prologue développe avec beaucoup de détails pour la transmission du Livre de Sydrac. Suit le récit qui explique comment Japhet aurait appris l'astronomie : un jour, en gardant ses moutons à la montagne, il aurait perdu son plus jeune fils, qu'il ne put retrouver pendant sept jours et douze heures ${ }^{30}$. Dieu aurait exaucé sa prière et envoyé un

Lucidaire (III 33 à III 89). Dans le corps du dialogue, les citations sont concentrées de nouveau entre les questions 464 (version courte 323) et 490 (version courte 347 ; citations tirées surtout de la fin du livre II et du début du livre III du Lucidaire) et entre 637 (version courte 481) et 666 (version courte 506 ; citations tirées surtout de la fin du livre I du Lucidaire). Voir, pour plus de détails, la liste des équivalences dans l'édition du Lucidaire de M. Türk, op. cit., p. 203-204.

28. Cette rubrique, citée d'après la transcription de l'éd. Holler, p. 130, ne se trouve que dans la version courte.

29. Éd. Holler, p. 153. Cette partie de l'introduction qui concerne la mise en écrit et la transmission de l'astrologie, est conçue d'après le modèle du premier prologue. Elle sera supprimée dans la version longue.

30. Ibid. Voir, dans le second prologue, l'épisode dans lequel Sydrac et Boctus vont chercher l'herbe qui défait l'enchantement de la tour « en une contree de la parfonde Ynde qui se nomme la Montaingne verte dou corbiau ", éd. Ruhe, p. 41-42, § 12-13 (cf. pour la version courte éd. Treanor, p. 54-55). 
ange pour lui enseigner l'art qui «sera appellee astronnomie» ${ }^{31}$. Comme l'avait prédit l'ange, Japhet aurait retrouvé son fils au bout de sept jours et douze heures. Le récit se termine avec l'explication allégorique des indications temporelles: les sept jours «si senefient les .vij. plannetes, et les .xij. heures senefient les .xij. singnes» du zodiaque ${ }^{32}$. La ressemblance avec la fin du second prologue est évidente : il se termine également avec l'explication de ce que "segnefient» en détail les matériaux avec lesquels Sydrac venait de pratiquer la lécanomantie ${ }^{33}$.

Avec l'introduction et le long traité qui suit arrive à son point culminant l'intérêt tout particulier de l'auteur pour l'astronomie qui a été préparé depuis le tout début de l'œuvre. Parmi les sciences dont Dieu aurait doté Sydrac, une seule est soulignée dans le premier prologue : 1'astronomie ${ }^{34}$. C'est pour sa perfection dans cet art que le roi Boctus fait venir le « phillosophe astronomien » ${ }^{35}$. Grâce à lui, il espère pouvoir résoudre le problème de la tour qui ne veut pas tenir debout, problème face auquel les « astronomiens » de son royaume avaient lamentablement échoué ${ }^{36}$. Au cours du dialogue, le roi questionne Sydrac à plusieurs reprises au sujet de cet art ${ }^{37}$, avant de poser une question plus générale, qui entraîne le long traité d'astrologie ${ }^{38}$.

31. Éd. Ruhe, p. 316 (cf. pour la version courte éd. Holler, p. 153). Voir, dans le second prologue, l'ange qui aurait appris à Sydrac comment provoquer par lécanomantie l'apparition de la Trinité au-dessus d'un «vaissel» rempli d'eau. Cette vision ébranle définitivement l'idolâtrie de Boctus, qui avait voulu voir Dieu « apertement » comme il peut voir ses idoles (éd. Ruhe, p. 44, § 23).

32. Éd. Ruhe, p. 316 (cf. pour la version courte éd. Holler, p. 154).

33. Éd. Ruhe, p. 46, § 34 (cf. pour la version courte éd. Treanor, p. 67-68) : « Les .iij. çouches segnefient la Sainte Trinité [...]. Le vaissel seignefie le monde [...]. L'yaue dedenz seignefie le Fis de Dieu qui vendra en la Virge [...]. L'yaue que je jetai en les .iiij. cantons seignefie le Fis de Dieu qui sera batiziez en yaue [...]. Les .iiij. cantons senefient les .iiij. bons houmes evangelistres [...]».

34. «Et si ot la grace de Dieu de savoir [...] l'estronomie et du firmament et des planetes et des singnes et des estoilles et des ores et des poins [...]» (éd. Ruhe, p. 1, $\S 3$; pour la version courte cf. éd. Treanor p. 2-3).

35. Éd. Ruhe, p. 41, § 11 (cf. pour la version courte éd. Treanor, p. 54).

36. Éd. Ruhe, p. 39-40, § 2-6 (cf. pour la version courte éd. Treanor, p. 49-51).

37. Voir p. ex. les questions 236 (version courte 153, éd. Treanor, p. 181-185), 416 (version courte 283, éd. Holler, p. 23-24) et 583 (version courte 433, éd. Holler, p. 112-113).

38. Pour l'analyse de ce traité, voir les publications de D. Ruhe, « La roe d'astronomie. Le livre de Sidrac et les encyclopédies françaises du moyen âge », dans M. Picone éd., L'enciclopedismo medievale, Ravenna, 1994, p. 293-310 ; « Eschatologie und Astrologie. Zeitkonzeptionen im Livre de Sidrac », dans T. Ehlert éd., Zeitkonzeptionen Zeiterfahrung Zeitmessung. Stationen ihres Wandels vom 
Le bilan est net : tel qu'il se présente dans sa structure d'ensemble aux triples encadrements, comme dans celle du dernier quart de l'œuvre, ordonné et systématique, le Livre de Sydrac ne mérite guère les reproches qui lui sont faits à propos de son « désordre » et du fait qu'il serait un dialogue « dénué [...] de tout plan réfléchi » ${ }^{39}$. Il ne les mérite ni quant à la construction fictive de l'ensemble du texte ni quant à la structure du dialogue propre qui, pour se donner un cadre, s'appuie sur le plan du Lucidaire, et renforce l'organisation systématique dans la partie finale, composée de traités.

La Fontaine de toutes sciences irrite parce qu'elle ne correspond pas aux attentes génériques face à un texte qui se veut encyclopédique. Comparé à 1'Image du monde de Gossouin de Metz, au Trésor de Brunetto Latini et au dialogue anonyme entre Placides et Timéo, pour nous en tenir aux seuls textes en langue vulgaire française, il est évident que l'auteur a voulu faire œuvre à part. En la faisant venir du fond des temps et des frontières du monde, il l'encadre d'une narration ambitieuse qui rapproche le héros éponyme d'une grande figure de prophète de l'Ancien Testament et lui attribue la plus haute descendance, garante de son omniscience. La forme dialogique dans laquelle est coulée l'exposition du savoir privilégie la liberté de quelqu'un qui, vivant en marge du monde, rencontre quelqu'un qui peut répondre à toutes les questions et se réjouit de pouvoir puiser à cette « fontaine de toutes sciences ». La structure de l'œuvre se veut le reflet de cette genèse : elle est organisée de façon systématique dans des parties importantes (l'encadrement théologique, la partie finale) et plus ou moins relâchée dans les autres. Comme ces dernières constituent les trois quarts de l'œuvre, l'impression de chaos, d'un chaos qui paraissait total, a frustré les chercheurs. L'idée d'un « désordre " prémédité, il est vrai, a de quoi surprendre dans le monde des textes encyclopédiques. Le succès extraordinaire de l'œuvre trois siècles durant montre que les lecteurs de l'époque ont su l'apprécier.

\section{L'ordre et le désordre : les deux versions}

Des structures apparemment contradictoires sont combinées dans le Livre de Sydrac. Que l'œuvre ait été conçue sous cette forme, est confirmé par une analyse comparative des deux versions du texte ${ }^{40}$. Pour en venir

Mittelalter bis zur Moderne, Paderborn, 1998, p. 203-22 ; « La divination au moyen âge. Théories et pratiques », dans R. Trachsler éd., Moult obscures paroles. Études sur la prophétie médiévale, Paris, 2007, p. 17-28.

39. Y. Lefèvre, op. cit., p. 324.

40. Comme le proposait déjà Robert Marichal (« Les traductions provençales du Livre de Sidrach précédées d'un classement des manuscrits français », dans École 
directement au résultat le plus important pour notre propos : si la version longue double le volume de l'œuvre, elle le fait en répartissant équitablement les ajouts. Un bloc de presque trois cents questions et réponses grossit la partie librement agencée du dialogue, intercalé juste avant le début du traité astrologique (questions 667-959), et c'est dans une proportion comparable qu'a été enrichi ce traité. La volonté de garder à la structure d'ensemble son équilibre spécifique est évidente.

D'autres détails le confirment : le bloc des 300 questions est encadré par des parties systématiques ; il débute par un extrait d'une bonne vingtaine de chapitres du Miroir du Monde (671-698) et se termine par un extrait d'une trentaine de chapitres du Secré des Secrés (926-956), concernant la physiognomonie et la diététique ${ }^{41}$. D'autre part, l'aspect dialogique de l'œuvre est souligné par l'introduction d'une centaine de questions intercalées çà et là dans la première partie (1-666), isolément ou par petits groupes de deux ou trois jusqu'à plus de vingt ${ }^{42}$.

Grossir le volume du Livre dans de telles dimensions aurait pu créer des problèmes pour son utilisation. La solution que présente la version longue pour éviter ce risque est la subdivision des réponses, qui sont réparties sur des questions nouvelles : dans quelques dizaines de cas, soixante-dix en tout, leur nombre est multiplié par deux, par trois et par quatre ${ }^{43}$. Les

$\overline{\text { nationale des Chartes : Positions des thèses, Paris, 1927, p. 79-82, ici p. 80), nous }}$ pensons que les deux versions, composées vers la fin du XIII ${ }^{\mathrm{e}}$ siècle, sont selon toute probabilité l'œuvre du même auteur et que la version courte a précédé la version longue.

41. Voir B. Wins, « Le Livre de Sidrac — Stand der Forschung und neue Ergebnisse », dans H. Brunner-N. R. Wolf, op. cit., p. 36-52, ici p. 50-51.

42. Il s'agit des questions 8, 29-34, 41, 49-52, 106-128, 158-166, 180, 217, 233235, 237-241, 286, 295, 308, 312-316, 331-335, 350-352, 364, 380, 389-391, 407, 417-418, 449, 518, 520, 525, 563, 613, 616, 627-634, 651.

43. Il s'agit des questions suivantes, dont les réponses en formaient une seule dans la version courte (entre parenthèses les numéros de l'éd. Treanor et Holler) : 6-7 (6), 10-11(8), 12-13 (9), 14-15 (10), 16-19 (11), 20-21 (12), 24-25 (15), 27-28 (17), 36-37 (19), 38-39 (20), 54-55 (38), 58-59 (41), 65-66 (47), 175-176 (le numéro manque dans le manuscrit, éd. Treanor, p. 152), 183-184 (119), 185-186 (120), 194-195 (127), 196-197 (128), 199-200 (130), 204-205 (134), 206-208 (135), 211-212 (138), 214-215 (140), 219-220 (143), 221-222 (144), 226-227 (148), 229-230 (150), 259-260 (171), 266-267 (177), 271-272 (181), 273-274 (182), 279-280 (187), 282-283 (189), 291-292 (196), 300-301 (203), 302-303 (204), 305-307 (206), 310-311 ( 208), 317-318 (209), $322-323$ (213), 340-341 (225), 345-346 (229), 361-362 (241), 367-368 (248), 374-375 (254), 386-387 (264), 392-393 (266), 398-399 (271), 403-404 (275), 405-406 (276), 410-411 (279), 412-413 (280), 419-420 (284), 423-424 (287), 425-426 (288), 446-448 (308), 469-470 (328), 480-481 (338), 492-493 (349), 497-498 (353), 546-547 (397), 609-610 (458), 645-646 (489), 648-650 (491), 1141-1142 (583), 1149-1152 (590), 1153-1158 (591), 1177-1178 (605), 1184-1185 (611). 
conséquences sont bien plus radicales pour le traité d'astrologie. Dans la première version du Livre, la présentation sous forme de bloc en soulignait le caractère exceptionnel : le traité n'était pas rythmé par les questions du roi, mais se présentait comme un tout, interrompu une seule fois par Boctus ${ }^{44}$. Ces deux questions sont maintenant subdivisées en cent cinq questions (960-1064). Dans le texte ainsi compartimenté, il était non seulement plus facile de trouver le chapitre auquel on s'intéressait, en parcourant les longues colonnes sur lesquelles s'étend le texte du traité (une centaine) ${ }^{45}$, mais il était aussi beaucoup plus aisé de s'orienter dans la table numérotée des questions au début du Livre : parmi les 105 rubriques (au lieu de deux dans la version courte), formulées suivant le même modèle, on pouvait désormais rapidement repérer la planète qu'on cherchait pour interpréter l'information qu'avait livrée la « roe de l'astronomie », et passer, grâce au numéro indiqué, directement au chapitre en question.

Face à l'amplification massive qui caractérise la seconde version, le nombre de questions qui sont éliminées est minime (7) ; elles concernent toutes des recettes de médecine, que la version longue enrichit, pour compenser, de dix nouvelles ${ }^{46}$. Bien plus intéressants sont les passages qui ont été supprimés çà et là dans le Livre dans environ soixante réponses. Les plus importants par leur étendue sont ceux dans lesquels avaient été résumées les informations bibliques concernant l'Ancien et le Nouveau Testaments :

$636 \mathrm{Li}$ quel seront ceulz qui naistront de Noe jusques a la venue du vrai prophete et seront amis de Dieu?

657 Quelle vertu fera le Filz Dieu en terre?

659 Les menistres du Filz Dieu porront il sanoir les emfermes aussi comme il fera?

La longue réponse à la question 636 (version courte 480) fait allusion à une cinquantaine de personnages surtout de l'Ancien Testament, à commencer par Melchisedec « dou quel Dieu digna reçoivre pain et vin [a] sacrefice » jusqu'aux rois mages ; la réponse à la question 657 (version

44. Version courte, question 507 (960-969 de la version longue), question 508 (970-1064 de la version longue).

45. F. 109rb-137rb dans le ms. de Londres (BL, Add. 17914), sur lequel est basée notre édition. Dans la version courte, l'astrologie en occupe moins de la moitié : par ex., dans le ms. Sainte-Geneviève 2202, f. 124ra-138ra.

46. Questions 462, éd. Holler, p. 124 ; 464-465, éd. Holler, p. 125 ; 468-469, éd. Holler, p. 126-127 ; 477-478 (la deuxième partie de la réponse à la question 477 constitue une nouvelle question : «Et se la chalor est as papilles [...]» etc.), éd. Holler, p. 129. Pour les dix nouvelles questions introduites dans la version longue, voir 613,616 et $627-634$. 
courte 497) énumère une trentaine de miracles de Jésus, celle à la question 659 (version courte 499) évoque une dizaine de cas de malades que les apôtres « saneront ». Toutes ces informations, condensées chacune en une phrase ${ }^{47}$, sont radicalement réduites dans la version longue.

Une première raison de ces coupures se laisse aisément deviner : c'est le souci d'augmenter la vraisemblance des 'prophéties' de Sydrac, à laquelle l'exposition de trop de connaissances détaillées concernant l'avenir du personnel biblique risquait de nuire. C'est ce que confirme la longue réponse à la question $636:$ de tous les personnages qui « naistront » depuis Noé n'est conservé que le premier («Melchisedech»), pour la simple raison que sa naissance ne devait plus être prophétisée, puisqu'il était déjà mort du temps de Sydrac ${ }^{48}$. La petite remarque qu'ajoute la seconde version avant ce nom ( « de nous avant nasquirent ») et qui est reprise après qu'a été cité ce nom, est explicite à ce sujet (« cestui fu avant de nous »). De la longue liste de tous les autres qui « naistront » et pour lesquels les noms avaient été introduits dans la version courte, ou bien comme gloses ou bien directement intégrés dans le texte, accompagnés quelquefois de la formule « qui avera non » ${ }^{49}$, de tout cela il ne reste qu'une phrase qui résume brièvement les mérites de ces « amis de Dieu ». Les réponses aux deux autres questions sont raccourcies dans les mêmes dimensions.

La dernière phrase de la réponse 659 ajoute un autre argument à la réduction radicale des très nombreux miracles des apôtres : « trop seroient ennuieuses a conter ». Ce désir de ne pas vouloir lasser le lecteur peut expliquer la suppression d'un bon nombre de passages, à commencer par trois longs extraits du Lucidaire. La question 479 «Quelle chose est emfer et comment $\mathrm{i}$ vont les ames?» provoque de longues explications qui culminent dans l'énumération des neuf peines de l'enfer, suivie de descriptions drastiques ${ }^{50}$ que la version longue ne reprend pas. Dans les réponses aux questions concernant Jésus (640 « Ou converssera le Filz Dieu quant il sera emfant?», 642 «Pour quoi mourra il et pour quoi se lera il morir ? ») ${ }^{51}$ n'est conservé que le noyau de l'énoncé. La tendance à réduire

47. D'après nos premiers sondages, elles ont été formulées sur la base des sommaires qui accompagnaient la Bible à l'époque.

48. L'auteur inconnu réussit à ne pas trahir la construction de sa fiction, qui l'oblige à employer constamment le futur pour parler du temps après Sydrac. Voir, p. ex., la question 635 « Les quieulx furent les amis de Dieu, d'Adam enjusques a Noé ? » et la question 1188 « Quans aages sont ? », dans laquelle il distingue entre les deux premiers âges qui furent jusques a Abraham, et le tiers qui «sera de Abraham jusques a Moyses ». Voir, de même, la question $644:$ : [...] Enoch, qui fu avant de Noe [...]».

49. Voir, p. ex., le ms. Sainte-Geneviève 2202, f. $117 \mathrm{rb}$.

50. Question 337 de la version courte, éd. Holler, p. 55-58, ici 57-58.

51. Dans la version courte, questions 484 et 486, éd. Holler, p. 135-136 et p. 137. 
les passages qui insistent trop sur l'aspect édifiant ou sur une interprétation compliquée se confirme souvent, tout d'abord par la suppression de formulations plus ou moins répétitives et de reprises d'idées en conclusion d'une réponse ${ }^{52}$, jusqu'aux comparaisons, largement développées quelquefois, qui sont éliminées ${ }^{53}$. La plus longue, soulignant la réponse à la question 61 dans la version courte ( Puet le mavais home avoir l'amor de Dieu come il bon ?»), évoquait l'histoire de gens qui font naufrage et se trouvent sur la rive face à deux ponts pour traverser deux fleuves; au-delà de l'un, un « riche homme » les exhorte à venir vers lui — il « tient mult de vestimens entour lui et si est assis [en] un bel jardin » - et les met en garde contre l'autre pont, qui serait fragile et les ferait tomber entre les mains de « jehans [...] a grans grafions » qui les jetteraient dans un grand feu. Tous les éléments de ce récit sont ensuite expliqués selon leur « senefiance » ${ }^{54}$.

Une fois n'est pas coutume : ce qui est supprimé dans un certain nombre de cas peut être conservé dans d'autres, comme c'est le cas pour la comparaison non moins longue qu'on trouve dans la réponse à la question $425^{55}$ ou pour l'énumération des douze apparitions de Jésus après sa résurrection ${ }^{56}$; et ce qui semble être complètement éliminé, peut réapparaître en partie ailleurs, retravaillé et intégré dans une autre réponse ou largement développé sous forme de nouvelles questions, ce qui témoigne du soin avec lequel la version longue a été élaborée ${ }^{57}$.

52. Quelques exemples: 132 (80 de l'éd. Treanor, p. 131); 134 (82 de l'éd. Treanor, p. 132) ; 142 (90 de l'éd. Treanor, p. 139) ; 246 (158 de l'éd. Treanor, p. 188); 321 (212 de l'éd. Treanor, p. 230).

53. Voir 64 de la version longue (46 de l'éd. Treanor, p. 105) : « ausi com uns chevaliers [...]»; 105 (76 de l'éd. Treanor, p. 121-122) : « Ensement votre grant secreut nus ne le puet savoir [...]. Ensement est dou secret de Dieu ». Dans la comparaison entre le secret de Dieu et celui d'un roi, Boctus est apostrophé directement en tant que roi ; 144 (92 de l'éd. Treanor, p. 141-142) : « ensi come .ii. homes qui alaissent en .ii. chemins [...]. Autresi est en cest siecle [...]. »; 147 (95 de l'éd. Treanor, p. 144-145) : « Li riche si est ensi com un vaisseil de terre [...]. Ausi sont les gens en cest siecle [...]. »; 148 (96 de l'éd. Treanor, p. 145) : « ausi come celui qui puet aler seurement en un plain et en petit chemin [...]»; 173 (sans numéro, éd. Treanor, p. 151) : « ausi com uns hons alast gaignier en une longaine terre [...] $) ; 221$ (144 de l'éd. Treanor, p. 173-174) : « Un rois disoit a un povre home [...] »; 421 (285 de l'éd. Holler, p. 25) : « autretel comme .ii. homes qui vont par .i. chemin [...]».

54. Éd. Treanor, p. 114-116, ici p. 115. La question 61 de la version courte correspond à la question 90 de la version longue.

55. Question 425 (288 de la version courte, éd. Holler, p. 27-28: « [...] ensement come .ii. homes d'un seignor qui sont en .i. cité, [...]. Ensement est de cels de Paradis et d'enfer ; [...]. »).

56. Question 644 (488 de la version courte, éd. Holler, p. 138-139).

57. Il est plus difficile de faire ce constat pour les très rares déplacements de questions dans la version longue. Dans les trois cas pour lesquels une question isolée 
La question 479 en est un bon exemple. Elle était formée dans la version courte (337) de longs extraits du Lucidaire (III 12-22), dont la deuxième partie est supprimée dans la version longue ${ }^{58}$. Mais à y regarder de près, on découvre que deux éléments ont été réemployés ailleurs : d'une part, l'évocation de la joie qu'éprouvent les justes quand ils voient « les maus homes tormenter » en enfer, même s'il s'agit de leurs plus proches parents ${ }^{59}$, est rattachée à la question 63 , dans laquelle le même passage du Lucidaire III 20 avait déjà été cité, mais sans la phrase qui y est incorporée maintenant; d'autre part, l'idée que « les justes ames » ne prient pas « pour ceuls qui sont en emfer » (voir Lucidaire III 21) est placée à la fin de la réponse 481, où elle sert de contrepoint à l'argumentation qui repose sur les questions précédentes du Lucidaire (III 24-26).

Le remaniement réorganise le texte en supprimant des passages qui faisaient double emploi. Il peut aussi le restructurer pour transformer une vague règle en un catalogue systématique et détaillé de conseils pratiques. La question qui concerne « les plus perilleuses colles du cors » (378) était suivi encore, dans la version courte, d'un passage qui, pour aborder le problème du rapport entre les "colles » et les quatre saisons, parlait surtout de calendrier, de planètes et de chiffres, avant d'arriver à la conclusion suivante : «Et par la nature de ces .iiii. saisons chascuns se puet garder des contraires, et se il le fait il n'aura jamais nulle enfermeté au cors ${ }^{60}$. C'est tout un petit traité, réparti sur plusieurs chapitres (950-954) ${ }^{61}$ et concentré exclusivement sur la santé, qu'introduit la version longue entre le traité de physiologie et le

ou deux ont été déplacées, le nouvel ordre améliore la cohésion avec le contexte (1124 et 1125 initialement intercalées après 1120 et 526 après 517). La raison du transfert reste pourtant obscure pour les questions 69-77 qui se trouvaient initialement intégrées après la question 40 (21 de la version courte : elles y portaient les numéros 22-30). V. Minervini pensait à « uno spostamento di fogli » (« Schede sulla tradizione manoscritta del Livre de Sidrach », dans Annali dell'Istituto universitario orientale-sezione romanza, t. 19, 2 [1977], p. 539-570, ici p. 547), mais cela n'explique pas tout, car trois autres questions ont été déplacées également qui se trouvaient dans la version courte après la question 362 ( 241 de la version courte) : la question 242 est devenue la question 42 dans la version longue, 243 y apparaît comme numéro 67 et 244 comme numéro 596.

58. Éd. Holler, p. 55-58, ici 57-58; le passage supprimé commence p. 57, ligne 5 (« et por ce ardent dou feu d'enfer [...]»).

59. «[...] ja soit ce que li peres voie le fil ou li filz le pere, ou la mere la fille ou la fille la mere [...] », Lucidaire III 20, éd. M. Türk, op. cit., p. 365.

60. Question 257 de la version courte, éd. Holler, p. 6-7, ici p. 7.

61. Le chapitre précédent et les deux chapitres suivants sont aussi directement liés à la santé (949: «Comment ne pour quoy puet garder homme son cors en santé par l'aide de Dieu ? »; 955 : «Comment se puet l'en engraissier ? ; 956 : « Comment se puet l'en amaigrir?»). 
début de l'astrologie, pour spécifier en détail ce qu'il faut observer à chaque saison ${ }^{62}$.

La révision du texte a laissé des traces à différents endroits, concernant les contenus divers de l'œuvre. Le dénominateur commun est la volonté de préciser et d'enrichir l'information. Ainsi la réponse à la question de l'inégalité entre les hommes (292 «Por quoy ne sont les gens tous communal en cest siecle et en l'autre?»; version courte 196) est-elle complétée de deux raisons, dont la première insiste sur l'aspect astrologique — l'importance du moment de la naissance (" pour les heures et les poins en coi il naissent ») — et la seconde sur l'aspect social (si les gens étaient égaux, «nuls ne servist l'autre, et ainssint ne pourroient il mie vivre ») ${ }^{63}$. De même la relativité des phénomènes naturels, tels que l'apparence de la grandeur des étoiles ou des mouvements du soleil ${ }^{64}$, est-elle rendue plus facile à saisir; l'explication qui est introduite pour ces derniers est un autre exemple d'un passage déplacé - dans la version courte il terminait initialement la réponse 120 (version longue 186) ; transféré au numéro 318 (version courte 209), les chiffres indiqués pour la course du soleil et celle de son ombre « en terre » ont sensiblement changé : à « .iiij. dois en terre » ne correspondent plus «.m. lieus » au firmament, ${ }^{65}$ mais «.iiij. liues », ce qui établit une correspondance parfaite entre les chiffres, qui rappelle celle qu'on peut constater dans les passages allégoriques déjà cités ${ }^{66}$.

62. Du texte de la version courte ne sont conservés que quelques éléments descriptifs : le printemps, qui est également appelé « erbage », caractérisé de chaud et humide (950), comme l'été l'est de chaud et sec (951), l'automne de froid et sec (952) et l'hiver de froid et humide (953).

63. Voir, de même, la question 303 (version courte 204, éd. Treanor, p. 222) dans laquelle est précisé exactement combien de son avoir on doit laisser à ses enfants (un tiers), ou la question 362 (version courte 241, éd. Treanor, p. 250-251) dans laquelle la version longue ajoute aux trois raisons qui prouvent que ceux qui en Dieu croient " seront plus dignes que les angeles » une quatrième qui insiste, pour conclure, sur le contenu de la deuxième raison. De même est ajoutée à la recette contre le saignement de nez une troisième méthode (question 602 ; version courte 451, éd. Holler, p. 120-121), comme est ajouté systématiquement à toutes les recettes (1093-1141) ce que dans la version courte n'était dit que pour une seule (1111, dans la version courte 553, éd. Holler, p. 204) : l'endroit où trouver l'herbe en question.

64. Voir 311 dans la version longue (208 dans la version courte, éd. Treanor, p. 226-227) et 318 (209 dans la version courte, éd. Treanor, p. 228-229).

65. Éd. Treanor, p. 160.

66. Voir supra, p. 8, pour le second prologue et le prologue du traité d'astrologie. Corriger un détail peut aussi avoir eu pour cause le désir de mieux le motiver : dans la question 265 (version courte 176, éd. Treanor, p. 200), le nom de Japhet, inventeur du premier instrument, est remplacé dans la version longue par deux noms, celui de Tubal et de Tubachaim (ou Tubalchaim, suivant les manuscrits). Tubalchaim est 
Ce n'est que très prudemment qu'on peut essayer de tirer des conclusions à propos des observations que l'analyse comparative des deux versions permet de faire. La Fontaine de toutes sciences coule à grands flots. Il est difficile de ne pas se laisser entraîner par la grande diversité de courants thématiques qui traversent le texte et de ne pas perdre de vue l'ensemble, dans lequel sont noyés çà et là des passages que l'analyse doit réunir pour éviter des conclusions hâtives.

S'il est facile de définir en une formule ce qui a motivé l'élaboration de la seconde version, quant à l'aspect formel — la volonté d'amplifier —, il est impossible de le faire pour les modifications qui concernent le contenu de l'œuvre. Ce qui réunit les multiples interventions, c'est qu'elles ne changent pas l'esprit qui animait déjà la première version de l'œuvre. C'est une conception pragmatique de la vie, qui recommande de regarder un problème par deux fois, « esperituelment » et « corporellement 》. Et de privilégier au besoin ce dernier aspect. Doit-on, par exemple, se soucier des autres (552; version courte 402) ? « Esperituelment » oui, « mes corporelment tu ne doiz mie pensser que de toi ou des tiens ».

Cette façon spécifique d'approcher le monde serait le propre du philosophe : « celui qui est sages et enlettré et qui respont du tout ce que l'en li demande, cist est apelez philosophes. Et les philosophes sont apelés maistres du monde corporel, car autres genz pueent enseignier et aprendre » ${ }^{67}$. Sydrac le philosophe parle de son propre rôle. Veut-il par là également faire allusion à l'auteur anonyme du Livre? Que cet inconnu ait été un homme largement « enlettré » ne fait pas de doute. Que sa formation l'ait plutôt placé parmi les maîtres du savoir séculier que parmi ceux du savoir théologique, correspond bien à l'esprit de son œuvre et au goût de la fiction qui lui donne forme. Ce penchant pour le jeu de masques et un scénario savamment calculé a ajouté au plaisir des lecteurs de son temps, comme il tient encore en haleine les chercheurs du nôtre.

Tout ce qui a été entrepris depuis plus d'un siècle pour préciser, au moins dans ses contours, la culture du personnage énigmatique qui se cache derrière Sydrac, en identifiant les sources auxquelles il a puisé pour remplir sa Fontaine, n'a mené encore qu'à des réponses partielles, fragmentaires. Elles sont autant de « promesses de nouvelles questions".

cité dans la Bible comme inventeur de l'art de travailler l'airain et le fer (Gen 4, 22 : « [...] Tubalcain, qui fuit malleator et faber in cuncta opera aeris et ferri »); le nom de Tubal, qui était un des fils de Japhet (Gen 10,2) semble avoir été confondu avec Iubal, cité dans le verset précédent (Gen 4, 21 : «[...] Iubal: ipse fuit pater canentium cithara et organo $»$.

67. Question 547; dans la version courte (question 397, éd. Holler, p. 92-93, ici 93), la première phrase ne commence qu'avec les mots « Et cil qui respondent de ce que l'om lor demande $[\ldots] »$. 


\section{Postscriptum}

La tradition manuscrite déjà bien fournie du Livre de Sydrac continue de s'enrichir ${ }^{68}$. Cela n'est guère surprenant, vu le succès de l'œuvre à son époque. Mais ce qui est une véritable surprise, c'est qu'on puisse voir apparaître un manuscrit complètement ignoré jusqu'en 2000 et pourtant d'une qualité exceptionnelle, voire unique : avec vingt-sept miniatures, c'est de loin le plus richement illustré parmi les plus de soixante-dix exemplaires existants ${ }^{69}$.

La surprise a rapidement fait place à la déception, car l'apparition n'a duré que le temps d'une vente aux enchères : elle a eu lieu le 5 décembre 2000 chez Sotheby's, à Londres, où le codex a changé de propriétaire pour la somme de 553500 livres. À peine sorti du néant, il y est rentré, disparaissant vers une destination gardée secrète ${ }^{70}$. Mais telle une étoile filante qui, en se vaporisant, libère une certaine luminosité, le manuscrit a laissé une trace, car une description en avait été faite pour préparer la vente. Curieusement et comme pour doubler le sort du Livre, elle se présente sous deux versions, une version assez courte, mais enrichie de deux illustrations du manuscrit, et une analyse plus fouillée. Toutes les deux ont été rendues accessibles en ligne et le sont restées jusqu'à aujourd'hui (janvier 2011) ${ }^{71}$.

68. Il s'agit de deux textes complets de la version courte (Cambridge, Fitzwilliam Museum, McClean 176 et Lincoln, Cathedral Libr. 212) et de deux fragments ; le premier a été publié par C. Connochie-Bourgne : « Un fragment inédit du Livre de Sydrach : sept feuilles d'une collection privée », dans Qui tant savoit d'engin et d'art. Mélanges de philologie ... Gabriel Bianciotto, Civilisation Médiévale, t. 16 (2006), p. 399-413 ; quant à l'autre, un fragment de Padoue, c'est G. Peron qui en prépare la publication (« Frammenti padovani del Livre de Sidrac [Padova, Bibl. del Seminario Vescovile, cod. 692] »).

69. Les autres manuscrits illustrés, quinze en tout, en présentent beaucoup moins. Nous les citons dans l'ordre descendant : 10 miniatures (Montpellier, Bibl. interuniv., Méd. H 149 ; Paris, BNF, fr. 1159) ; 9 (Lyon 948) ; 6 (Londres, BL, Harley 4417 ; Paris, BNF, fr. 1161) ; 4 (Paris, BNF, fr. 762) ; 3 (Londres, BL, Add 16563 ; Paris, BNF, fr. 1157) ; 2 (Amsterdam 116 ; Londres, BL, 17914 ; Bruxelles 11106, 11113 ; Paris, BNF, fr. 24395) ; 1 (Londres, BL, Royal 16.F.V ; s'Gravenhage 133 A 2).

70. L'accueillante formule "Contact us » sur le site de Sotheby's ne s'adresse visiblement pas aux chercheurs : nos lettres demandant plus d'informations sur le manuscrit sont restées sans réponse ; cela semble être l'usage de la maison, comme nous avons pu le constater pour d'autres ventes dans le passé.

71. Pour la version courte voir: http://files.shareholder.com/downloads/-BID/ 0x0x103171/-8e3d0926-f2af-4e2a-a607-31bad8227261/20001030-26968.pdf. Les illustrations reproduites sont celles qui accompagnent les questions 185 (les 
Que le manuscrit ait été complètement inconnu s'explique par son histoire. Entré dans la bibliothèque du tsar, acheté « from the spoils of the French Revolution », il avait été mis en vente dans les années trente par le gouvernement soviétique et acquis par le collectionneur bibliophile Marcel Jeanson; ses héritiers l'ont remis sur le marché au seuil du $\mathrm{XXI}^{\mathrm{e}}$ siècle.

D'après l'analyse de Sotheby's, le manuscrit, témoin de la version courte du Livre de Sydrac, daterait des toutes premières années du XIV ${ }^{\mathrm{e}}$ siècle et aurait été exécuté à Arras. Les miniatures seraient l'œuvre de l'artiste du manuscrit Paris, BNF, lat. 1328, qui a illustré aussi d'autres manuscrits célèbres pour leur qualité. Le vaste programme qu'elles constituent n'a que peu de choses en commun avec d'autres manuscrits illustrés, parmi lesquels les deux manuscrits le plus richement décorés, témoins également de la version courte, montrent le plus de parenté ${ }^{72}$. Vu le soin exceptionnel avec lequel le manuscrit a été écrit et peint, il reste à espérer qu'il sera généreusement rendu accessible à un futur éditeur de la version courte ${ }^{73}$.

Ernstpeter RUHE

Université de Würzburg

antipodes ; le même thème est illustré dans le ms. Paris, BNF, fr. 1161, f. 55ra) et 211 (l'arche de Noé ; le même thème est illustré dans les mss Londres, BL, Harley 4417, f. 46rb ; Lyon 948, f. 133vb ; Montpellier H 149, f. 55v et f. 108r [question 494]; Paris, BNF, fr. 1159, f. 59vb). Pour la version plus fouillée voir http://www.sothebys.com/app/live/lot/LotDetail.jsp?lot_id=39XNR.

72. Il s'agit des manuscrits Montpellier H 149 et Paris, BNF, fr. 1159.

73. D'après sa publication toute récente Le domande del principe. Piccole enciclopedie dialogiche romanze, Milano, 2009, L. Sacchi semble avoir fait quelques pas importants qui pourraient préparer une telle entreprise. Nous venons de découvrir ce livre, dans lequel l'auteur consacre un long chapitre au Livre de Sydrac (p. 115-173). Que cette œuvre volumineuse soit classée sous le titre de «Petites encyclopédies » a de quoi surprendre. L'étude de Sacchi, basée sur quatre textes en langue romane (que le titre du livre n'indique pas : il s'agit, en dehors du Sydrac, de la Summa de philosophia in vulgari, du Placides et Timéo et du Lucidario di Sancho IV) a pour double but d'analyser « i contorni comuni e le strategie » de ces œuvres et leurs " somiglianze e differenze con gli antecedenti latini » (p. 18, c'est le sujet traité dans l'introduction) ainsi que les transformations de chacune d'elles (ibid.). Sacchi reconstruit avec beaucoup de soin ces transformations pour les deux versions du Livre de Sydrac à travers la complexe tradition manuscrite de cette œuvre. 